\title{
Medical case management after laminectomy or craniotomy: do all patients benefit from admission to the intensive care unit?
}

\begin{abstract}
James A. Nitahara, M.D., Malou Valencia, M.S.N., and Michael A. Bronstein, M.D.
Departments of Internal Medicine and Nursing, Kaiser Permanente Medical Center, Los Angeles, California
\end{abstract}

To define severity of illness to identify most effectively patients for whom admission to the intensive care unit (ICU) is unnecessary, the authors performed a retrospective cost-effectiveness analysis. The authors studied the records of 113 patients who were admitted to the ICU after undergoing laminectomy (or other spinal cord surgery) or craniotomy for removal of neoplasm; the Acute Physiology and Chronic Health Evaluation III prognostic system had identified these patients as having a $10 \%$ or less risk of requiring intervention while in the ICU. No patient required active intervention during a mean stay of 3.26 days in the ICU. Combined use of a "step-down" postoperative care unit and ICU can optimize allocation of medical resources while providing high-quality care for some neurosurgical patients who are at low risk of requiring postoperative intervention.

Key Words * APACHE (Acute Physiology and Chronic Health Evaluation)* craniotomy * laminectomy * low-risk monitor

With the advent of sophisticated data collection systems such as the Acute Physiology and Chronic Health Evaluation (APACHE), the Pediatric Risk of Mortality, and the McGill Pain Map systems, allocation of medical resources has come under close scrutiny. New technologies and increasing costs cause intensive care medicine to drain hospital resources. The cost of critical care beds continues to account for an increasing portion of hospital expenditures.[12] Although specific monitoring and intervention certainly require the degree of care delivered in the intensive care unit (ICU) setting, clinical assessment must now be supplemented by objective criteria to justify admission to the ICU. These criteria could include a definition of severity of illness as it relates to level of care required and medical outcome, which would be helpful in identifying patients for whom admission to the ICU is unnecessary. At our hospital, we use the APACHE III prognostic system to assess ICU utilization.

Many patients who have undergone neurosurgical procedures are admitted postoperatively to our ICU solely for nursing care and hourly neurological evaluation. This protocol is followed in many institutions to assure prompt intervention for potential postoperative complications. [8] We incorporated the APACHE III prognostic system to investigate outcomes for patients admitted to the ICU under this 
rationale after having undergone laminectomy or craniotomy. Our goal was to observe the clinical course in patients who have a less than $10 \%$ risk of requiring active medical or surgical intervention while in the hospital. We used the APACHE III protocol to determine the degree to which these patients received ICU intervention as an indication of appropriate ICU admission. Our results showed that the APACHE III prognostic system accurately predicted which patients could be safely and effectively treated in a non-ICU setting and helped us to more efficiently allocate ICU resources.

\section{CLINICAL MATERIAL AND METHODS}

Kaiser Foundation Hospital in Los Angeles is a 595-bed tertiary care center that serves the Los Angeles metropolitan area and has an 18-bed medical/surgical ICU. The ICU is run by a full-time intensivist who oversees one medical resident, one surgical resident, and two medical interns. In addition, practitioners of various medical and surgical subspecialties actively participate in patient care. The hospital's critical care committee has developed written guidelines for level of care provided in the ICU. Although the ICU is not a "closed unit," the intensivist in charge has authority to deny admission to the ICU. Nurses specializing in critical care provide nursing care, and the nurse/patient ratio is 1:1 or 1:2, depending on patient requirements. All patients admitted to the ICU are observed and have their vital signs monitored at least hourly.

In 1996, 1471 patients were admitted to the ICU; 301 (20.46\%) of these patients were admitted after undergoing neurosurgical intervention (cardiac and cardiac surgical patients are admitted to a separate ICU dedicated to their care) and all critically ill adult patients are admitted to the medical/surgical ICU. We retrospectively reviewed all admissions to the ICU between January 1 and December 31 of 1996 . We used the APACHE III prognostic system to identify all patients who were admitted after being treated neurosurgically. The APACHE III prognostic system, described elsewhere,[5] predicts mortality risk for critically ill patients admitted to the hospital.

We investigated profiles for admitted patients classified by APACHE III as having undergone laminectomy/other spinal cord surgery or craniotomy for neoplasm resection. The laminectomy category included all lumbar as well as cervical laminectomy procedures, with or without fusion, and discectomy procedures. The craniotomy category included all craniotomy procedures related to removal of neoplasm. We evaluated the records of 113 patients within these categories identified by APACHE III criteria as "low-risk monitor" (LRM) patients (that is, patients who have a less than $10 \%$ risk of needing active medical or surgical intervention such as intubation or dialysis) while in the ICU. No distinction was made between cervical and lumbar laminectomy because no complications occurred for any patient who underwent these procedures. The APACHE III data we examined included information on gender, age, length of stay (LOS), APACHE III score, Glasgow Coma Scale (GCS) score, need for ICU active intervention, ICU mortality, and ICU readmissions. The APACHE III score represents the sum of three groups of variables (physiology, age, and chronic health problems). The type of intervention required for one patient was determined by chart review. The charts of two patients requiring readmission to the ICU were reviewed to determine why.

\section{RESULTS}

Of the 1471 patients admitted to the ICU during the study period, 301 had undergone a neurosurgical procedure. Of this group, 64 patients were admitted after undergoing laminectomy and 67 patients were admitted after undergoing craniotomy for removal of a neoplasm. The number of LRM patients was 57 $(89.1 \%)$ in the laminectomy group and $56(83.6 \%)$ in the craniotomy group. The characteristics of each 
LRM population are reviewed in Table 1. The mean age of the LRM patients who underwent laminectomy was 58.9 years; 21 were men and 36 were women. Ethnic distribution was noted to include 27 Caucasian, 15 African American, eight Hispanic, and seven Asian patients. The mean length of ICU stay was 2.8 days; the mean acute physiology score was 16.4; the mean APACHE III score was 23.3; and mean GCS score was 14.9.

\begin{tabular}{|c|c|c|}
\hline $\begin{array}{l}\text { Th } \\
\text { CHARACTERISTICS OF } 113 \text { PATIENTS AD } \\
\text { AND CONSDERED AT LOW RISK FOR }\end{array}$ & $\begin{array}{l}\text { TO THE ICU AI } \\
\text { IG POSTOPERA }\end{array}$ & $\begin{array}{l}\text { NEUROSURGERY } \\
\text { INTERYENTION }\end{array}$ \\
\hline & Neurosurg & Tocedure \\
\hline Characteristic & $\begin{array}{l}\text { Laminectomy } \\
\text { (57 patibents) }\end{array}$ & $\begin{array}{l}\text { Cranidomy } \\
\text { (56 patients) }\end{array}$ \\
\hline mean age in yrs & $\begin{array}{r}58.94 \\
(t+15.5)\end{array}$ & $\begin{array}{r}54.04 \\
(+144)\end{array}$ \\
\hline $\operatorname{sex}$ & $21 \mathrm{M}$ & $20 \mathrm{M}$ \\
\hline race & & \\
\hline Caucasian & 27 & 32 \\
\hline African American & 15 & 17 \\
\hline Hispanic & 8 & 7 \\
\hline Asian & 7 & 0 \\
\hline acute ph ysiology score & 16.4 & 19.17 \\
\hline APACHE III soore & 23.3 & 26.87 \\
\hline GCS soore & 14.9 & 14.3 \\
\hline length of ICU stay (days) & 2.8 & 3.5 \\
\hline obser wed to predicted length of stay & 1.1 & 1.4 \\
\hline
\end{tabular}

The mean age of patients in the craniotomy group was 54 years; 20 were men and 36 were women. There were 32 Caucasian, 17 African American, and seven Hispanic patients. The mean length of ICU stay was 3.5 days; the mean acute physiology score was 19.17; the mean APACHE III score was 26.87; and the mean GCS score was 14.3.

The ratio of observed to predicted LOS for the two groups of patients was 1.1 for the laminectomy group and 1.4 for the craniotomy group. No patient in either group died during the hospital stay. One patient in the craniotomy group was readmitted to the ICU after having a grand mal seizure in the hospital unit. Two patients in the laminectomy group were readmitted to the ICU from the hospital unit for prescheduled follow-up surgical procedures.

\section{DISCUSSION}

Critical care patients have higher mortality rates and consume approximately four times the resources compared with patients treated on a standard-floor care nursing unit. From our APACHE III data, we were able to assess medical outcomes and carefully scrutinize resources allocated to delivery of critical care.

We searched the medical literature for previous studies regarding postoperative care and mortality rates of patients who have undergone laminectomy or craniotomy. Most neurosurgical patients are admitted to an ICU postoperatively for close observation by nursing staff and critical care monitoring. Patients who have undergone cervical laminectomy are admitted to an ICU primarily for observation of signs of spinal cord or tracheal compression caused by bleeding. In a study by Knaus, et al.,[4] only $15 \%$ of all neurosurgical patients admitted to an ICU postoperatively received active treatment. Teplick, et al.,[11] 
reviewed the records of 263 surgical patients to assess efficacy of delivering initial postoperative care in the ICU and found that of 13 patients who had undergone cervical laminectomy, none required active treatment while in the ICU. In addition, Teplick, et al.,[11] wrote that patients who undergo elective craniotomy had infrequent postoperative complications and could be observed safely for 4 hours in a recovery-room setting before being transferred to a neurosurgical unit.

Before the advent of computer data collection, no consistent way to predict postoperative neurosurgical complication was available except by clinical assessment and GCS score. Using this measure in 1992, Koonsman, et al.,[6] claimed that patients admitted to the hospital with a diagnosis of basilar skull fracture and who had a GCS score less than 13 without intracranial disease did not need intensive care monitoring. Physicians' predictions of mortality do not vary much from the APACHE prediction.[1] The most common reasons for ICU readmission are that patients experience cardiopulmonary insufficiency and infection.[9] Erring on the side of caution is wiser because readmission to the ICU increases the risk of mortality to greater than $25 \%$. However, when the clinician has reproducible data from a group of similar patients, risk becomes simpler to evaluate.

Mean LOS in the ICU was 3.26 days for both groups in our study. Our data indicated that each patient could have been safely transferred from the unit after 24 hours. This policy would have reduced the number of ICU days by 255 and thus might have reduced overall hospital stay. Mean APACHE III score was 23.3 for patients in the laminectomy group and 26.87 for patients in the craniotomy group. When the APACHE III scores are less than or equal to 20, the relative importance of disease is small;[5] this was confirmed by our results, which showed that the low-risk patients were admitted to the ICU for observation and nursing care.

Shorter overall hospital stays may be related to physicians' reluctance to discharge patients directly from the ICU. The implication for hospital costs is obvious. In addition, rationing ICU services is a pertinent issue during bed shortages.[10] These low-risk neurosurgical patients have been objectively identified by APACHE III as potential candidates for early transfer so that ICU beds will be available for patients who have more severe illness.

At our institution and many others, all patients who undergo postsurgical laminectomy and elective craniotomy are admitted to the ICU postoperatively for frequent neurological checks. As the cost of ICU care increases, specialized step-down units have become more cost effective and are increasingly being instituted.[13] Based on analysis of our data, LRM patients with these diagnoses could have been cared for in such a step-down unit. A postoperative step-down unit can provide the same monitoring and patient support with a higher ratio of patients to nurses than in the traditional ICU.[10] In addition, patients can be chosen through a combination of clinical assessment and objective prediction.[14]

Previous studies have shown a short-stay unit created specifically for patients who underwent laminectomy and discectomy resulted in shorter hospital stay and no associated increase in complication rate.[7] However, most of the studies in which postoperative neurosurgical care was described were done in the last 10 years and before integration of data collection systems was widely validated. Most step-down units emphasize respiratory care because it is generally the primary impetus for readmission to the ICU.[2] The step-down unit may need to be more fully developed to incorporate these low-risk surgical patients.

At our institution, we met with neurosurgical and nursing staff to devise a postoperative care plan to enable LRM patients who underwent laminectomy to go directly to a step-down unit to receive vital sign 
and neurological checks every 2 hours. The LRM patients who underwent craniotomy would be monitored in the ICU for 24 hours and then go to a step-down unit. This protocol is recommended by neurosurgical texts.[3] Since January 1, 1997, we have been monitoring mortality and readmission rates. To date, examination of our results has shown that the step-down unit is an effective and cost-sparing method of maintaining high-quality postoperative care for patients who have undergone laminectomy or craniotomy.

\section{Acknowledgment}

The authors thank the Medical Editing Department at the Kaiser Foundation Research Institute for providing editorial assistance.

\section{References}

1. Chang RWS, Jacobs S, Lee B, et al: Predicting deaths among intensive care unit patients. Crit Care Med 16:34-42, 1988

2. Durbin CG Jr, Kopel RF: A case-control study of patients readmitted to the intensive care unit. Crit Care Med 21:1547-1553, 1993

3. Kelly DF: Neurosurgical postoperative care. Neurosurg Clin North Am 5:789-810, 1994

4. Knaus WA, Draper E, Lawrence DE, et al: Neurosurgical admissions to the intensive care unit: intensive monitoring versus intensive therapy. Neurosurgery 8:438-442, 1981

5. Knaus WA, Wagner DP, Draper EA, et al: The APACHE III prognostic system. Risk prediction of hospital mortality for critically ill hospitalized adults. Chest 100:1619-1636, 1991

6. Koonsman M, Dunn E, Hughes K, et al: How much monitoring is needed for basilar skull fractures? Am J Surg 164:487-490, 1992

7. Lubkey TB, Fox R, Petruk KC, et al: Dedicated short-stay spinal unit. Axone 16:93-95, 1995

8. Ropper AH, Kennedy SK: Postoperative neurosurgical care, in Ropper AH (ed): Neurological and Neurosurgical Intensive Care, ed 3. New York: Raven Press, 1993, pp 185-191

9. Snow N, Bergin KT, Horrigan TP: Readmission of patients to the surgical intensive care unit: patient profiles and possibilities of prevention. Crit Care Med 13:961-964, 1985

10. Strauss MJ, LoGerfo JP, Yeltatzie JA, et al: Rationing of intensive care unit services. An everyday occurrence. JAMA 255:1143-1146, 1986

11. Teplick R, Caldera DL, Gilbert JP, et al: Benefit of elective intensive care admission after certain operations. Anesth Analg 62:572-577, 1983

12. Wagner DP, Knaus WA, Draper EA, et al: Identification of low-risk monitor patients within a medical-surgical intensive care unit. Med Care 21:425-434, 1983

13. Zilm F, Brown ML: Evaluating ICU beds and the use of step-down units. Healthcare Financ Manage 38:26-27, 32-34, 1984 
14. Zimmerman JE, Wagner DP, Knaus WA, et al: The use of risk predictions to identify candidates for intermediate care units. Implications for intensive care utilization and cost. Chest 108:490-499, 1995

Manuscript received April 2, 1998.

Accepted in final form July 10, 1998.

Presented at the 5th annual meeting of the APACHE Medical Systems, Inc., User Group Meeting, San Diego, California, April 18, 1997, and published in abstract form in the proceedings.

Address reprint requests to: Michael A. Bronstein, M.D., Department of Internal Medicine, Kaiser Permanente Medical Center, 4950 Sunset Boulevard, Atrium Level, Los Angeles, California $90027-6021$. 